\title{
MAXIMAL IDEALS IN LAURENT POLYNOMIAL RINGS
}

\author{
BUDH NASHIER
}

(Communicated by Louis J. Ratliff, Jr.)

\begin{abstract}
We prove, among other results, that the one-dimensional local domain $A$ is Henselian if and only if for every maximal ideal $M$ in the Laurent polynomial ring $A\left[T, T^{-1}\right]$, either $M \cap A[T]$ or $M \cap A\left[T^{-1}\right]$ is a maximal ideal. The discrete valuation ring $A$ is Henselian if and only if every pseudoWeierstrass polynomial in $A[T]$ is Weierstrass. We apply our results to the complete intersection problem for maximal ideals in regular Laurent polynomial rings.
\end{abstract}

\section{INTRODUCTION}

Let $A$ be a commutative Noetherian ring with identity. Let $R$ denote the Laurent polynomial ring $A\left[X_{1}, \ldots, X_{n}, Y_{1}, Y_{1}^{-1}, \ldots, Y_{m}, Y_{m}^{-1}\right]$, where $X_{i}$ and $Y_{i}$ are distinct indeterminates over $A$. Let $M$ be a maximal ideal in $R$. Let

$$
M_{1}=M \cap A\left[X_{1}, \ldots, X_{n}, Y_{1}, \ldots, Y_{m}\right]
$$

and

$$
M_{2}=M \cap A\left[X_{1}, \ldots, X_{n}, Y_{1}^{-1}, \ldots, Y_{m}^{-1}\right] .
$$

The content of this paper is the investigation of the following question.

Question. When is $M_{1}$ or $M_{2}$ a maximal ideal? In other words, when do maximal ideals in $R$ come from maximal ideals in $A\left[X_{1}, \ldots, X_{n}, Y_{1}, \ldots, Y_{m}\right]$ or $A\left[X_{1}, \ldots, X_{n}, Y_{1}^{-1}, \ldots, Y_{m}^{-1}\right]$ ?

We provide a complete answer to this question. With the above setup of notations, we prove that for every maximal ideal $M$ in $R, M_{1}$ or $M_{2}$ is a maximal ideal if an only if $A / P$ is a Henselian ring for every $G$-ideal $P$ in $A$. As a consequence, we prove that the one-dimensional local domain $A$ is Henselian if and only if for every maximal ideal $M$ in the Laurent polynomial ring $A\left[T, T^{-1}\right]$, either $M \cap A[T]$ or $M \cap A\left[T^{-1}\right]$ is a maximal ideal, and thus we answer a question suggested in [12, Remark, p. 689].

Since a quotient of a Henselian ring is Henselian, it follows that if $A$ is Henselian then for every maximal ideal $M$ in $R$, either $M_{1}$ or $M_{2}$ is maximal. Abhyankar, Heinzer, and Wiegand [1] have produced an example of a nonHenselian ring $A$ such that $A / P$ is Henselian for every $G$-ideal $P$ in $A$.

Received by the editors October 5, 1990 and, in revised form, January 10, 1991.

1980 Mathematics Subject Classification (1985 Revision). Primary 13B25, 13F20, 13J15. 
For terminology my standard source is Nagata [10]. All the rings we consider are commutative Noetherian with identity. The dimension of a ring means the Krull dimension, and all rings are assumed to have finite dimension.

\section{Preliminaries}

Let us recall that in the ring $A$ a prime ideal $P$ is called a $G$-ideal if $P$ is the contraction of a maximal ideal in the polynomial ring $A[T]$ (see [8]). It is well known (and is easy to prove) that a prime ideal $P$ in $A$ is a $G$-ideal if and only if $A / P$ is a semilocal domain of dimension $\leq 1$. The ring $A$ is, by definition, a Hilbert ring if every $G$-ideal in $A$ is maximal. Finitely generated algebras over Hilbert rings are Hilbert rings. Thus, in the case when $A$ is a Hilbert ring, we observe that both $M_{1}$ and $M_{2}$ are maximal ideals.

Let $P=M \cap A$. A generalized version of a theorem of Artin and Tate [2, Theorem 4] amounts to the following: If $B$ is a Noetherian domain such that some finitely generated $B$-algebra is a semilocal domain of dimension $\leq 1$, then $B$ is semilocal of dimension $\leq 1$ For a proof of this statement, see [6, 15.1]. In the situation under consideration, we have that the field $R / M$ is a finitely generated $(A / P)$-algebra, so we conclude that $A / P$ is a semilocal domain of dimension $\leq 1$. Hence $P$ is a $G$-ideal.

The following couple of theorems are crucial to the proofs of our main results.

Theorem A [12, Theorem 2.2]. Let $A$ be a local domain of dimension one. Then $A$ is Henselian if and only if $A^{\prime}$ (the derived normal ring of $A$ ) is a discrete valuation ring such that if $f \in A^{\prime}[T]$ is an irreducible polynomial of degree $\geq 1$, then either $f$ is monic or $f(0)$ is a unit in $A^{\prime}$.

Lacking a proper reference, I choose to give a proof of the following needed result. Recall that a ring $A$ is said to satisfy the first chain condition for prime ideals if every maximal chain of prime ideals in $A$ has length equal to the dimension of $A[10$, p. 123].

Theorem B. Let $A$ be a Noetherian domain of dimension $d$. Assume that $A$ satisfies the first chain condition for prime ideals. Let $R$ be the Laurent polynomial ring $A\left[X_{1}, \ldots, X_{n}, Y_{1}, Y_{1}^{-1}, \ldots, Y_{m}, Y_{m}^{-1}\right]$. Then the height of every maximal ideal in $R$ is $d+n+m$ or $d+n+m-1$.

Proof. Let $M$ be a maximal ideal in $R$ and $P=M \cap A$. If $P$ is a maximal ideal in $A$, then $M / P R$ is a maximal ideal in the affine domain $(A / P)\left[X_{1}, \ldots, X_{n}, Y_{1}, Y_{1}^{-1}, \ldots, Y_{m}, Y_{m}^{-1}\right]$ over the field $A / P$. Hence $\operatorname{ht}(M / P R)=n+m$. By assumption, $\operatorname{ht}(P)=d$. Thus it follows that $M$ has height $d+n+m$. If $P$ is not maximal then $\operatorname{dim}(A / P)=1$. By the assumption that $A$ satisfies the first chain condition for prime ideals, we have ht $(P)=d-1$. $M / P R$ is a maximal ideal in the domain $(A / P)\left[X_{1}, \ldots, X_{n}, Y_{1}, Y_{1}^{-1}, \ldots\right.$, $\left.Y_{m}, Y_{m}^{-1}\right]$ such that $(M / P R) \cap(A / P)=(0)$. Going through the quotient field of $A / P$, we observe that ht $(M / P R)=n+m$. Then, ht $(M) \geq \operatorname{ht}(P)+\operatorname{ht}(M / P R)=$ $d-1+n+m$. On the other hand, let us observe that for any prime ideal $Q$ in $R, \operatorname{ht}(Q) \leq \mathrm{ht}(Q \cap A)+n+m$. Hence $\operatorname{ht}(M)=d-1+n+m$. 


\section{The MAIN RESUlTS}

We first prove a simple lemma.

Lemma 1. Let $A \rightarrow B$ be an integral extension of domains. Then for every maximal ideal $M$ in the ring $A\left[X_{1}, \ldots, X_{n}, Y_{1}, Y_{1}^{-1}, \ldots, Y_{m}, Y_{m}^{-1}\right]$, either $M_{1}=$ $M \cap A\left[X_{1}, \ldots, X_{n}, Y_{1}, \ldots, Y_{m}\right]$ or $M_{2}=M \cap A\left[X_{1}, \ldots, X_{n}, Y_{1}^{-1}, \ldots, Y_{m}^{-1}\right]$ is a maximal ideal if and only if for every maximal ideal $N$ in the ring $B\left[X_{1}, \ldots, X_{n}, Y_{1}, Y_{1}^{-1}, \ldots, Y_{m}, Y_{m}^{-1}\right]$, either $N_{1}=N \cap B\left[X_{1}, \ldots, X_{n}, Y_{1}\right.$, $\left.\ldots, Y_{m}\right]$ or $N_{2}=N \cap B\left[X_{1}, \ldots, X_{n}, Y_{1}^{-1}, \ldots, Y_{m}^{-1}\right]$ is a maximal ideal.

Proof. We only prove one part leaving the other for the reader. Let us assume that either $M_{1}$ or $M_{2}$ is maximal for every maximal ideal $M$. Let $N$ be a maximal ideal in $B\left[X_{1}, \ldots, X_{n}, Y_{1}, Y_{1}^{-1}, \ldots, Y_{m}, Y_{m}^{-1}\right]$. Let $M=N \cap$ $A\left[X_{1}, \ldots, X_{n}, Y_{1}, Y_{1}^{-1}, \ldots, Y_{m}, Y_{m}^{-1}\right]$. Without loss of generality, we assume that $M_{1}=M \cap A\left[X_{1}, \ldots, X_{n}, Y_{1}, \ldots, Y_{m}\right]$ is a maximal ideal. Let $N_{1}=$ $N \cap B\left[X_{1}, \ldots, X_{n}, Y_{1}, \ldots, Y_{m}\right]$. We show that $N_{1}$ is a maximal ideal. Observe $M_{1}=N_{1} \cap A\left[X_{1}, \ldots, X_{n}, Y_{1}, \ldots, Y_{m}\right]$. Since $B\left[X_{1}, \ldots, X_{n}, Y_{1}, \ldots, Y_{m}\right]$ is integral over $A\left[X_{1}, \ldots, X_{n}, Y_{1}, \ldots, Y_{m}\right]$ and the prime ideal $N_{1}$ contracts to the maximal ideal $M_{1}$ of $A\left[X_{1}, \ldots, X_{n}, Y_{1}, \ldots, Y_{m}\right]$, we have that $N_{1}$ is maximal.

We now prove

Theorem 1. Let $A$ be a ring such that $A / P$ is a Henselian ring for every $G$-ideal $P$ in $A$. Let $M$ be a maximal ideal in the Laurent polynomial ring $R=A\left[X_{1}, \ldots, X_{n}, Y_{1}, Y_{1}^{-1}, \ldots, Y_{m}, Y_{m}^{-1}\right]$. Then either $M_{1}=M \cap$ $A\left[X_{1}, \ldots, X_{n}, Y_{1}, \ldots, Y_{m}\right]$ or $M_{2}=M \cap A\left[X_{1}, \ldots, X_{n}, Y_{1}^{-1}, \ldots, Y_{m}^{-1}\right]$ is a maximal ideal.

Proof. Let $P=M \cap A$. The first reduction is that we go modulo $P$, and assume that $M \cap A=(0)$. Then $A$ is a semilocal domain of dimension $\leq 1$. If $\operatorname{dim}(A)=0$ then $A$ is a field, and we have that both $M_{1}$ and $M_{2}$ are maximal ideals. So, we assume that $\operatorname{dim}(A)=1$. Using the given hypothesis, we have that $A$ is a Henselian local domain.

We now proceed by induction on $m$. Let $A^{\prime}$ denote the derived normal ring of $A$. Since $A$ is a Henselian local domain of dimension one, $A^{\prime}$ is a Henselian discrete valuation ring. By Lemma 1 , we may pass onto $A^{\prime}$ to prove the theorem. Thus, we assume that $A$ is a Henselian discrete valuation ring with $\pi \in A$ as a uniformizing parameter. By virtue of Theorem $\mathrm{B}$, we have that $\operatorname{ht}(M)=1+n+m$ or $n+m$. Since $M \cap A=(0)$, it is the case that $\operatorname{ht}(M)=n+m$. We note that $\operatorname{ht}\left(M_{1}\right)=\operatorname{ht}\left(M_{2}\right)=n+m$. Set $Y=Y_{1}$. Let $Q=M \cap A[Y]=M_{1} \cap A[Y]$. Then $Q$ is a prime ideal of height one in $A[Y]$, and $M_{1} / Q$ is a prime ideal of height $n+m-1$ in the polynomial ring $(A[Y] / Q)\left[X_{1}, \ldots, X_{n}, Y_{2}, \ldots, Y_{m}\right]$.

If $Q$ is a maximal ideal then we observe that $M_{1} / Q$ is a maximal ideal. Hence $M_{1}$ is a maximal ideal. Suppose that $Q$ is not a maximal ideal. Since $A[Y]$ is a unique factorization domain, we have that $Q=(f)$, where $f$ is an irreducible polynomial in $A[Y]$. By Theorem A, either $f$ is monic or $f(0)$ is a unit in $A$. Changing $A[Y]$ to $A\left[Y^{-1}\right]$, if necessary, there is no loss of generality in assuming that $f$ is a monic polynomial in $A[Y]$.

Case (i). $f(0) \in \pi A$. Let $f=Y^{t}+a_{1} Y^{t-1}+\cdots+a_{t}$. Since $f$ is an 
irreducible monic polynomial in $A[Y]$ with $a_{t}=f(0) \in \pi A$ and $(A, \pi)$ is a Henselian discrete valuation ring, we must have that each $a_{i} \in \pi A$; otherwise $f$ modulo $\pi A$ would factor as a product of two comaximal monics yielding a nontrivial factorization of $f$ in $A[Y]$. Let $Q^{\prime}=M \cap A\left[Y^{-1}\right]$. Then $Q^{\prime}$ is generated by $g=1+a_{1} T+\cdots+a_{t} T^{t}$, where $T=Y^{-1}$. At this point, we make the observation that $g$ is not contained in any maximal ideal of height two in $A\left[Y^{-1}\right]$; this is because any maximal ideal of height two in $A\left[Y^{-1}\right]$ contains $\pi$, and thus it is co-maximal to $g$. Thus we have that $Q^{\prime}$ is a maximal ideal in $A\left[Y^{-1}\right]$. By an argument similar to the one given earlier, we conclude that $M_{2}$ is a maximal ideal.

Case (ii). $f(0) \notin \pi A$. Then $(f, Y)=A[Y]$, and $f$ is monic in $Y$. Hence we have that the integral domain $A_{1}=A[Y] / f A[Y]=A\left[Y, Y^{-1}\right] / f A\left[Y, Y^{-1}\right]$ is an integral extension of $A$. Since $A$ is a Henselian domain of dimension one, we have that $A_{1}$ is a Henselian domain of dimension one [10,43.13]. We go modulo $f A\left[Y, Y^{-1}\right]$ and obtain that $M^{\prime}=M / f A\left[Y, Y^{-1}\right]$ is a maximal ideal in $A_{1}\left[X_{1}, \ldots, X_{n}, Y_{2}, Y_{2}^{-1}, \ldots, Y_{m}, Y_{m}^{-1}\right]$. By induction on $m$, either $M_{3}=$ $M^{\prime} \cap A_{1}\left[X_{1}, \ldots, X_{n}, Y_{2}, \ldots, Y_{m}\right]$ or $M_{4}=M^{\prime} \cap A_{1}\left[X_{1}, \ldots, X_{n}, Y_{2}^{-1}, \ldots, Y_{m}^{-1}\right]$ is a maximal ideal.

Let us note that $M_{1} / f A[Y]=M_{1} / Q=M_{3}$ and $M_{4}=M_{2} / Q^{\prime}$. Consequently, $M_{1}$ or $M_{2}$ is a maximal ideal. The proof is complete.

To establish the converse of Theorem 1, we need the following lemma.

Lemma 2. Let $A$ be a one-dimensional semilocal domain. Assume that for every maximal ideal $M$ in the Laurent polynomial ring $A\left[T, T^{-1}\right]$, either $M \cap A[T]$ or $M \cap A\left[T^{-1}\right]$ is a maximal ideal. Then the derived normal ring of $A$ is local; in particular, $A$ is local.

Proof. Let us assume that $A^{\prime}$, the derived normal ring of $A$, is not local. Since $A$ is a one-dimensional Noetherian semilocal domain, $A^{\prime}$ is a semilocal Dedekind domain $[10,33.2,33.10]$. Hence $A^{\prime}$ is a principal ideal domain with only a finite number of prime ideals $[14, \mathrm{p} .12]$. Let $p_{1}, p_{2}, \ldots, p_{r}$ be all the distinct (nonassociate) primes in $A^{\prime}$. By assumption we have $r>1$. Let $a_{0}=$ $p_{1} p_{2} \cdots p_{r}$. For each $i=1,2, \ldots, r$, let $a_{i}=p_{1} p_{2} \cdots p_{i-1} p_{i+1} \cdots p_{r}$. In the polynomial ring $A^{\prime}[T]$, set $f=a_{0}+a_{1} T+\cdots+a_{r} T^{r}$. By Eisenstein's criterion, $f$ is an irreducible polynomial in $A^{\prime}[T]$. Since $f A^{\prime}[T]$ is not a maximal ideal, any maximal ideal in $A^{\prime}[T]$ containing $f A^{\prime}[T]$ will have height two. Then such a maximal ideal must contain some $p_{i}$ and hence $T$. So the maximal ideals in $A^{\prime}[T]$ that contain $f A^{\prime}[T]$ are precisely $\left(p_{i}, T\right), i=1,2, \ldots, r$. Hence $M=f A^{\prime}\left[T, T^{-1}\right]$ is a maximal ideal in $A^{\prime}\left[T, T^{-1}\right]$, and we have that neither $M \cap A^{\prime}[T]$ nor $M \cap A^{\prime}\left[T^{-1}\right]$ is a maximal ideal. By letting $P=M \cap A\left[T, T^{-1}\right]$, we observe that $P$ is a maximal ideal in $A\left[T, T^{-1}\right]$ such that its contractions to $A[T]$ and $A\left[T^{-1}\right]$ are not maximal, a contradiction. Hence $A^{\prime}$ is local.

We now prove

Theorem 2. Let $A$ be a ring such that for every maximal ideal $M$ in the Laurent polynomial ring $A\left[X_{1}, \ldots, X_{n}, Y_{1}, Y_{1}^{-1}, \ldots, Y_{m}, Y_{m}^{-1}\right]$, either $M_{1}=M \cap$ $A\left[X_{1}, \ldots, X_{n}, Y_{1}, \ldots, Y_{m}\right]$ or $M_{2}=M \cap A\left[X_{1}, \ldots, X_{n}, Y_{1}^{-1}, \ldots, Y_{m}^{-1}\right]$ is a maximal ideal. Then $A / P$ is a Henselian ring for every $G$-ideal $P$ in $A$.

Proof. Let $P$ be a $G$-ideal in $A$. If $P$ is maximal then $A / P$ is trivially 
Henselian. So we assume that $P$ is not maximal. Then $A / P$ is a one-dimensional semilocal domain. Note that the hypothesis in the statement of the theorem remains valid when we replace $A$ by $A / P$ (pay attention to only the maximal ideals containing $P$ ). So we replace $A$ by $A / P$ and then prove that $A$ is Henselian. By Lemma $2, A$ is local. To prove that $A$ is Henselian, it suffices to prove that every domain $B$ that is an integral extension of $A$ is quasilocal [10, 43.12]. This is equivalent to proving that any domain $B$ that is a finite $A$-module is local. Let $B$ be a domain that is a finite $A$-module. By Lemma $1, B$ enjoys the hypothesis assumed for $A$. By Lemma $2, B$ is local. Hence $A$ is Henselian.

Since the zero ideal is a $G$-ideal in a local domain of dimension one, we have

Corollary 1. Let $A$ be a one-dimensional local domain. Then $A$ is Henselian if and only if every maximal ideal in the Laurent polynomial ring $A\left[T, T^{-1}\right]$ contracts to a maximal ideal in $A[T]$ or to a maximal ideal in $A\left[T^{-1}\right]$.

Heinzer, Lantz, and Wiegand have independently proved Corollary 1.

\section{Applications}

Let $(A, \mathscr{M})$ be a quasi-local ring. In [12] we defined a polynomial $f$ in $A[T]$ to be pseudo-Weierstrass if $(\mathscr{M}, T)$ is the only maximal ideal in $A[T]$ that contains $f$.

Let $(A, \mathscr{M})$ be a quasi-local ring. A monic polynomial $f \in A[T]$ is called a Weierstrass polynomial if $f=T^{n}+a_{1} T^{n-1}+\cdots+a_{n}$, where each $a_{i} \in \mathscr{M}$.

Clearly a Weierstrass polynomial is pseudo-Weierstrass. If $A$ is a local domain of dimension at least two, then pseudo-Weierstrass polynomials in $A[T]$ are precisely the Weierstrass ones, as was proved in [12, Proposition 3.1]. Let $(A, \pi)$ be a discrete valuation ring such that the polynomial $f=\pi T^{2}+T+\pi$ is irreducible in the polynomial ring $A[T]$. Then $(\pi, T)$ is the only maximal ideal in $A[T]$ that contains $f$. Hence $f$ is a pseudo-Weierstrass polynomial that is not Weierstrass. So, in the case of a discrete valuation ring, when is every pseudo-Weierstrass polynomial Weierstrass? In [12, Theorem 3.6] it was proved that if $A$ is a discrete valuation ring, then every pseudo-Weierstrass polynomial in $A[T]$ is Weierstrass if and only if every maximal ideal in the Laurent polynomial ring $A\left[T, T^{-1}\right]$ contracts to a maximal ideal in $A[T]$ or in $A\left[T^{-1}\right]$. Thus a combination of Corollary 1 and [12, Theorem 3.6] gives us the following.

A1. Let $A$ be a discrete valuation ring. Then every pseudo-Weierstrass polynomial in $A[T]$ is Weierstrass if and only if $A$ is Henselian.

Let $I$ be an ideal in a ring $R$. We say that $I$ is a complete intersection ideal if it can be generated as an $R$-module by $\mathrm{ht}(I)$ elements. A Noetherian ring $R$ is called strongly regular if every maximal ideal of $R$ is a complete intersection ideal [6, p. 148]. In [5, Theorem 2] it was shown that a polynomial extension of a regular Hilbert domain is strongly regular. Thus we have

A2. A Laurent polynomial extension of regular Hilbert domain is strongly regular.

Regular Hilbert domains exist in abundance. The rings of polynomial functions on nonsingular algebraic varieties are classical examples of such domains. 
A really interesting way to get examples of regular Hilbert domains is the following. Start with a Noetherian ring $A$. Let $A[T]$ be the polynomial ring in one indeterminate over $A$, and let $A(T)$ denote the localization of $A[T]$ at the multiplicative set of all monic polynomials. Then $A(T)$ is a Hilbert ring. This result was the content of [4]. A very easy and nice way (following a suggestion of J. T. Stafford) to see this is as follows. Set $Y=1 / T$. Let $S$ be the multiplicative set $1+Y A[Y]$ in $A[Y]$. Throw $Y$ in the Jacobson radical by forming the ring $B=S^{-1} A[Y]$. It is easy to verify that $A(T)=B[1 / Y]$; for details, see [9, p. 99]. Now the fact that $A(T)$ is a Hilbert ring is a consequence of the following beautiful application [7, 10.5.8] of the Principal Ideal Theorem of Krull: Let $B$ be a Noetherian ring, and let $a$ be a nonnilpotent element in the Jacobson radical of $B$. Then $B[1 / a]$ is a Hilbert ring. Thus if we start with a regular ring $A$, then we have that $A(T)$ is a regular Hilbert domain. Let us now record the following.

A3. If $A$ is a regular ring then $A(T)\left[X_{1}, \ldots, X_{n}, Y_{1}, Y_{1}^{-1}, \ldots, Y_{m}, Y_{m}^{-1}\right]$, with $n+m \geq 1$, is a strongly regular ring.

For A3, if $A$ is a regular locality (localization at a regular prime ideal of an affine algebra over a field) with infinite residue field or if $A$ is a formal power series ring over a field, then $n+m$ may be zero; this was proved in [13]. It is not known whether $A(T)$ is a strongly regular ring for any regular local ring $A$.

Let $A$ be a Henselian local ring such that polynomial extensions of $A$ are strongly regular (consequently, $A$ is a regular local ring). Then using Theorem 1 , we have that Laurent polynomial extensions of $A$ are also strongly regular. For instance, it is known that if $A$ is a formal power series ring with coefficients in a field, then any polynomial extension of $A$ is a strongly regular ring [3, Theorem 3.1; 11, Theorem 2.2]. Thus, we have

A4 (cf. 15, Theorem 2.8). Let $A=k\left[\left[T_{1}, \ldots, T_{d}\right]\right]$, where $k$ is a field. Then the Laurent polynomial ring $A\left[X_{1}, \ldots, X_{n}, Y_{1}, Y_{1}^{-1}, \ldots, Y_{m}, Y_{m}^{-1}\right]$ is strongly regular.

\section{ACKNOWLEDGMENT}

I express my sincere thanks to Professor Sylvia Wiegand. Her interest in this work inspired me to obtain a complete answer to the question in the introduction.

\section{REFERENCES}

1. S. S. Abhyankar, W. Heinzer, and S. Wiegand, On the compositum of two power series rings Proc. Amer. Math. Soc. 112 (1991), 629-636.

2. E. Artin and J. Tate, A note on finite integral extensions, J. Math. Soc. Japan 3 (1951), 74-77.

3. S. M. Bhatwadekar, A note on complete intersections, Trans. Amer. Math. Soc. 270 (1982), 175-181.

4. J. W. Brewer and W. Heinzer, $R$ Noetherian implies $R\langle X\rangle$ is a Hilbert ring, J. Algebra 67 (1980), 204-209.

5. E. D. Davis and A. V. Geramita, Efficient generation of maximal ideals in polynomial rings, Trans. Amer. Math. Soc. 231 (1977), 497-505. 
6. A. V. Geramita and C. Small, Introduction to homological methods in commutative rings, 2nd ed., Queen's Papers in Pure and Appl. Math., vol. 43, Queen's Univ., Kingston, Canada, 1979.

7. A. Grothendieck, Éléments de géométrie algébrique. IV, Inst. Hautes Études Sci. Publ. Math. 28 (1966).

8. I. Kaplansky, Commutative rings, revised ed., Univ. of Chicago Press, Chicago, 1974.

9. T. Y. Lam, Serre's conjecture, Lecture Notes in Math., vol. 635, Springer-Verlag, Berlin, Heidelberg, and New York, 1978.

10. M. Nagata, Local rings, Interscience, New York, 1962.

11. B. Nashier, Efficient generation of ideals in polynomial rings, J. Algebra 85 (1983), 287-302.

12. _. Henselian rings and Weierstrass polynomials, Proc. Amer. Math. Soc. 112 (1991), 685-690.

13. __ Strongly regular rings, J. Algebra 137 (1991), 206-213.

14. J.-P. Serre, Local fields, Graduate Texts in Math., vol. 67, Springer-Verlag, Berlin, Heidelberg, and New York, 1979.

15. P. L. N. Varma, Efficient generation of zero dimensional ideals in polynomial and Laurent polynomial rings, Ph.D. dissertation, Univ. of Hyderabad, Hyderabad, 1990.

Department of Mathematics, Florida State University, Tallahasee, Florida 32306 